\title{
The Relation of Severe Malnutrition in Infancy to the Intelligence of School Children with Differing Life Histories
}

\author{
STEPHEN A. RICHARDSON ${ }^{25}$ \\ Departments of Pediatrics and Community Health, Rose F. Kennedy Center for Research in Mental Retardation and \\ Human Development, Albert Einstein College of Medicine, Bronx, New York, USA
}

\section{Extract}

The IQ's of Jamaican boys aged 6-10 were associated significantly with the presence or absence of severe malnutrition in infancy, with height at time of IQ testing, and with a measure of the boys' social background. A multiple correlation coefficient of 0.674 was obtained between IQ and the three factors. Social background contributed 0.294 of the variance, height 0.112 , and severe malnutrition 0.049. The two extreme groups of boys, i.e., those malnourrished, small at follow-up, and with unfavorable social backgrounds and those not malnourished, tall at follow-up, and with favorable social backgrounds had average IQ's of 49.4 and 74.9, respectively (from Table 5 ). Only two of the boys in the most advantaged group had IQ scores that overlapped with the most disadvantaged group. Boys with severe malnutrition in infancy, but who are tall at follow-up and have a favorable social background have an average IQ 11 points higher than boys who did not experience severe malnutrition, but who are short at followup and have a unfavorable social background. The difference in IQ between boys who did and did not experience severe malnutrition in infancy varies under different conditions of height and social background when those are held constant for both groups. Under the most favorable conditions of being tall and having an advantageous social history the average IQ of the malnourished boys is only 2 points lower than those not malnourished. Under the most unfavorable conditions of short stature and a disadvantageous social background the IQ of the malnourished boys is 9 points lower than those not malnourished (Table 6 and Fig. 1).

\section{Speculation}

The long range consequences of a severe episode of malnutrition in infancy can be understood only in the context of the human ecologic conditions including social, economic, and biologic factors which the child and his family have experienced during their lifetimes. Results of the present study suggest that an episode of severe malnutrition in infancy in the context of a lifetime of generally favorable experiences for child development does not appear to cause any intellectual impairment, but when severe malnutrition occurs in an ecology generally unfavorable for intellectual development, the early malnutrition has a clear relation to later intellectual impairment.

It is still not clear whether severe malnutrition in infancy causes intellectual impairment despite numerous studies which have examined the issue $(3-6,9-11,16,20)$. There is fairly general agreement that infants who have suffered severe malnutrition in infancy do less well at later ages on tests of learning and intelligence than do control subjects who were not malnourished. In human studies the matching procedures used for selecting control subjects have been inadequate to control many of the factors known or postulated to influence the intellectual development of children. The inability to obtain adequate controls suggests that it is necessary to examine an episode of severe malnutrition in infancy in the context of a child's overall life history and determine under what circumstances and conditions severe malnutrition in infancy is associated with mental impairment at older ages. The selection of which background factors in a child's history are chosen for study must be based on a consideration of what social and biologic factors influence intellectual development.

The purpose of this paper is to examine the ways in which intelligence of school age boys is related to the presence or absence of severe malnutrition in their infancy, their overall life history of nutrition, and the social and economic conditions they have experienced during their upbringing. This is one of a series of reports of a study of Jamaican boys (10, 12-18).

\section{SUBJECTS AND METHODS}

Seventy-four Jamaican boys were selected who had been treated in a hospital for severe malnutrition during their first 2 years of life (hereafter referred to as "index cases"). The malnutrition was reflected variously in syndromes of marasmus, kwashiorkor, or marasmic kwashiorkor. Evidence of the malnutrition was obtained from the detailed clinical and metabolic hospital records. These chitdren received an average of 8 weeks of inpatient care with good medical care and feeding of good quality and quantity. Follow-up visits were made by nurses to the boys' homes for 2 years after discharge. These boys were later traced and at the time of the intelligence test ranged in age from 6-10 years. These ages were selected so as to be far enough removed from the time of acute illness to eliminate the effects of immediate sequelae and for the boys to be at an age when intelligence testing has predictive value.

A classmate or neighbor comparison was selected for each index case. For index boys attending school a classmate nearest in age and of the same sex was chosen. Eleven of the 74 index boys were not attending school because of lack of school facilities. For these 11 cases a comparison subject was chosen by finding the nearest neighboring child who was not a relative, was of the same sex, and was within 6 months of age of the index case. It was determined from interviews with their parents or guardians that none of the comparison subjects had been hospitalized for severe malnutrition. Three comparison subjects were unavailable at the time of testing. This method of selection obtained 71 matched pairs of index and comparison cases who lived in the same general neighborhood. This made it unlikely that index and comparison matched cases would come from widely differing socioeconomic classes, but provided for variability of life experience among the study subjects within the same neighborhood.

Each child's intellectual level was individually evaluated by means of the WISC (22). Clearly, the IQ's obtained for Jamaican 
children are not directly comparable with those of children in the cultures where the test was standardized. However, as Vernon (21) has pointed out, comparisons of children within a culture on a test standardized in another setting are appropriate provided that the test discriminates between the individuals tested. Experienced testers were used who did not know whether a subject was an index or comparison case. A more detailed description of the selection of subjects and use of the WISC is given by Hertzig et al. (10).

To obtain data on the social background histories of the boys in the study and their families, a home interview was conducted with the mother or guardian. Additional information was obtained from observations made by the interviewer during the visit for the interview. The answers to selected questions or observations were combined to form a variable. Each social background history variable was hypothesized to be associated with intellectual development. From the 14 variables described by Richardson (13), 3 were selected which provide itiformation about the quardians, the economic conditions of the families, and the early experiences of the boys in the study (see Charts 1-3).

The indicators which together operationally define a variable are

Chart 1. Variable I: Caretaker's Level of Capability

\section{Indicator A}

Q. If you have or ever had any free time, what sort of things do you like to do most?

100-Religious or educational activities, games, sports, dancing, exercise, TV, movies, visiting, go on trips, read

0 -Sit, rest, sleep, smoke, or never has free time, must work all the time

Indicator B

Q. Do you read and write? If yes; can you read the Gleaner?

Q. Do you read any other newspaper?

100-Can read and write, reads any newspaper

0 -Cannot read or write, or can but does not read any newspaper

Indicator $\mathrm{C}$

Q. Are there people who come to see you sometimes and ask for your help or advice? If yes:

Q. What for?

100-People come for advice, for help with children or sickness, or for more than one reason

50-People come for one of the following reasons: help with cultivating, help with housework, to borrow money or food.

$0-$ No one comes

Indicator D: Interviewer rating based on observation

Q. On the whole, what was the state of neatness and organization in the house (e.g., yard swept, how well rooms kept up and arranged, any signs of excessive rubbish or dirt)?

100-Fairly well or very well organized

0 -Rather poorly or very poorly organized

Indicator $\mathrm{E}$ : Interviewer ratings

Q. How well did she understand the questions in the interview?

Q. How well did she respond to the questions?

Q. How would you rate her intelligence?

Q. Did she have any difficulty in thinking in terms of ideas?

100-Rated fairly well or better in understanding, fairly well or better in responding, average or better intelligence for the mothers interviewed, and some or no difficulty in thinking abstractly (i.e., rated fairly well or better on all four questions)

75-Rated fairly well or better on three out of four questions

$50-$ Rated fairly well or better on two out of four questions

25-Rated fairly well or better on one out of four questions

$0-$ Rated as having any difficulty in understanding, any difficulty in responding, below average in intelligence for the mothers interviewed, and as only being able to deal with concrete, matter-of-fact things (i.e., rated poorly on all four questions)
Chart 2. Variable II: Home furnishings and appliances

Q. Do you use electric power? If yes: What do you use electricity for?

Indicator $\mathrm{A}$

100-Three or more appliances using electricity

50 - One or two appliances using electricity

0 -No appliances

(These exclude use of refrigerator and sewing machine)

Indicator B

100-Presence of electric refrigerator

$0-$ No refrigerator

Indicator C: If no electricity, or if informant did not mention an electric sewing machine

Q. Do you have a sewing machine not run by electricity?

100 - Has a sewing machine

0 - Has no sewing machine

Indicator $\mathrm{D}$

Q. What fuel do you use for cooking?

100-Electricity, kerosene, bottled gas

$50-$ Coal

$0-$ Wood

Indicator $\mathrm{E}$

Q. Do you have a transistor radio?

$100-$ Yes

$0-\mathrm{No}_{0}$

Indicator $\mathrm{F}$

Q. How many beds do you have? (Answers were coded according to type of bed)

Number of persons in household derived from census questions

Person-bed ratio. Total number of persons in household divided by total number of bed spaces

100 -Less than 1.75 persons per bed

$0-1.75$ or more persons per bed

each scored on a scale of $0-100$. A 0 score is given to responses hypothesized to be the most disadvantageous for a child's intellectual development and a score of 100 is given to responses hypothesized to be most advantageous. Where indicators can be meaningfully classified into more than two categories, scores between 0 and 100 were assigned. This procedure gives each indicator an equal weight. To obtain a total score for the variable, the scores on each indicator are added and the sum divided by the number of indicators. The result gives a variable score which is an average of the indicator scores, and a range of $0-100$. To form an overall social background history variable, the scores on the three variables (Charts 1-3) were summed and these total scores were divided into deciles.

The episode of hospitalization for severe malnutrition provides a measure of nutrition for part of the first 2 years of life. To obtain a measure which provides some indication of the child's overall life history of nutrition, the height of the boys at the time of follow-up was used. Height has been used in previous studies of children living in poor areas where it was thought that malnutrition was common. Malnutrition has been inferred using a study design comparing the tall and short children $(7,8)$.

Other factors such as genetic differences also influence height, but in societies where stunting of growth occurs because of poor malnutrition, height will in part reflect nutrition. The height of each boy was obtained at the same time as the IQ tests were administered. To make the heights comparable across the age range of the subjects, standard scores were obtained based on standards developed for Jamaican primary school boys 7 years and older (1), and for rural Jamaican boys under 7 (2).

\section{RESULTS}

Severe malnutrition in infancy, height at follow-up, and social background history were examined separately for their association with IQ. The index boys, who had been hospitalized for severe 
Chart 3. Variable III: Intellectual stimulation

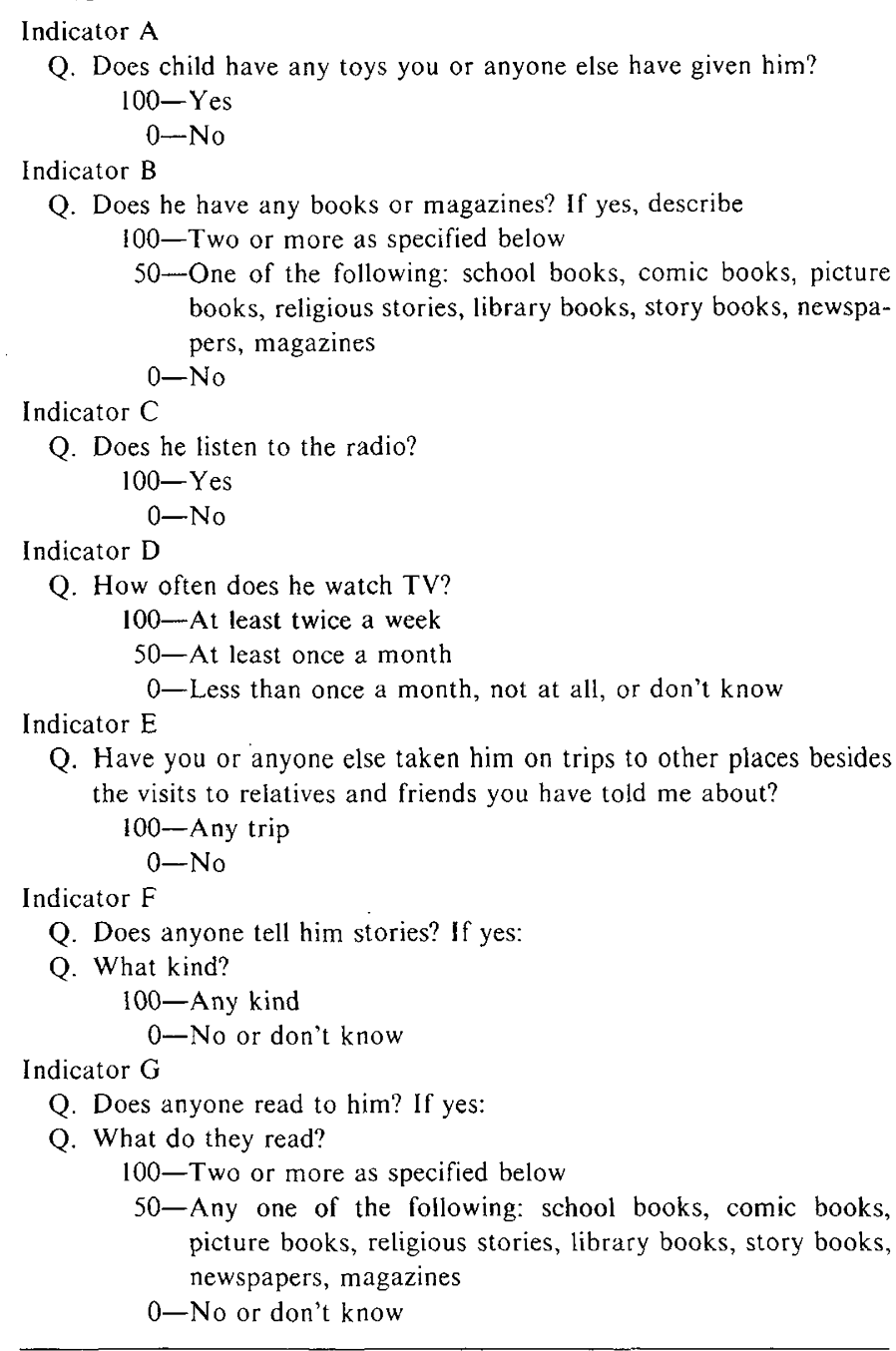

malnutrition during the first 2 years of life, had significantly lower IQ's than their matched comparison subjects, who had not been hospitalized (Table 1) (10).

To examine the relation between height at follow-up and IQ, the groups of index and comparison boys were combined. The distribution of heights was divided into three categories: tall, greater than $0.5 \mathrm{SD}$ above Jamaican mean height for boys; medium, $0.5 \mathrm{SD}$ above to $0.5 \mathrm{SD}$ below the mean; short, greater than $0.5 \mathrm{SD}$ below the mean height. The taller boys have IQ's significantly higher than the shorter boys (Table 2 ).

To examine the social background history of the boys and its association with IQ, the groups of index and comparison boys were again combined. Boys with the lower or more disadvantageous social background history scores had significantly lower IQ's than those with higher or more advantageous social background scores (Table 3).

Having shown that malnutrition in infancy, height at follow-up, and social background history are each associated with IQ in the expected direction, the next step was to determine the relative contribution of each of the three variables to IQ. To do this a multiple correlation coefficient was used. The overall coefficient is 0.674 , which, when squared, indicates that $46 \%$ of the variance is accounted for. When the overall variance is broken down into the three components, the largest contributor is the social background measure, which provides 0.294 of the variance. Hospitalization in infancy for severe malnutrition is the smallest contributor, with 0.049 of the variance, and height provides an intermediate variance of 0.112 (Table 4)
A somewhat different perspective of the results is obtained by examining the IQ's of the boys with different histories. First the IQ scores of the boys with the most disadvantageous and most advantageous histories were compared. Subset $l$ consisted of index boys who had been hospitalized for malnutrition, whose height was more than $0.5 \mathrm{SD}$ below the Jamaican mean $(1,2)$, and whose social background score was in the lowest 4 deciles. Subset 2 consisted of comparison boys not hospitalized for malnutrition, with heights more than $0.5 \mathrm{SD}$ above the Jamaican mean, and whose social background score was in the top 4 deciles. The IQ's of the boys in these two extreme subsets are strikingly different (Table 5). Half of the boys in subset 1 have IQ's on the floor of the

Table 1. Difference in $I Q$ of index and comparison boys

\begin{tabular}{lcc}
\hline & $\begin{array}{c}\text { Index } \\
(n=71)\end{array}$ & $\begin{array}{c}\text { Comparison } \\
(n=71)\end{array}$ \\
\hline $\begin{array}{l}\text { Full scale IQ } \\
\text { Mean }\end{array}$ & & \\
SD & 57.72 & 65.99 \\
Matched $t=5.25$ & 10.75 & 13.59 \\
$\quad P<0.001$ & & \\
\hline
\end{tabular}

${ }^{1}$ Data from Hertzig et al. (10).

Table 2. Index and comparison boys combined by $I Q$ and height ${ }^{1}$

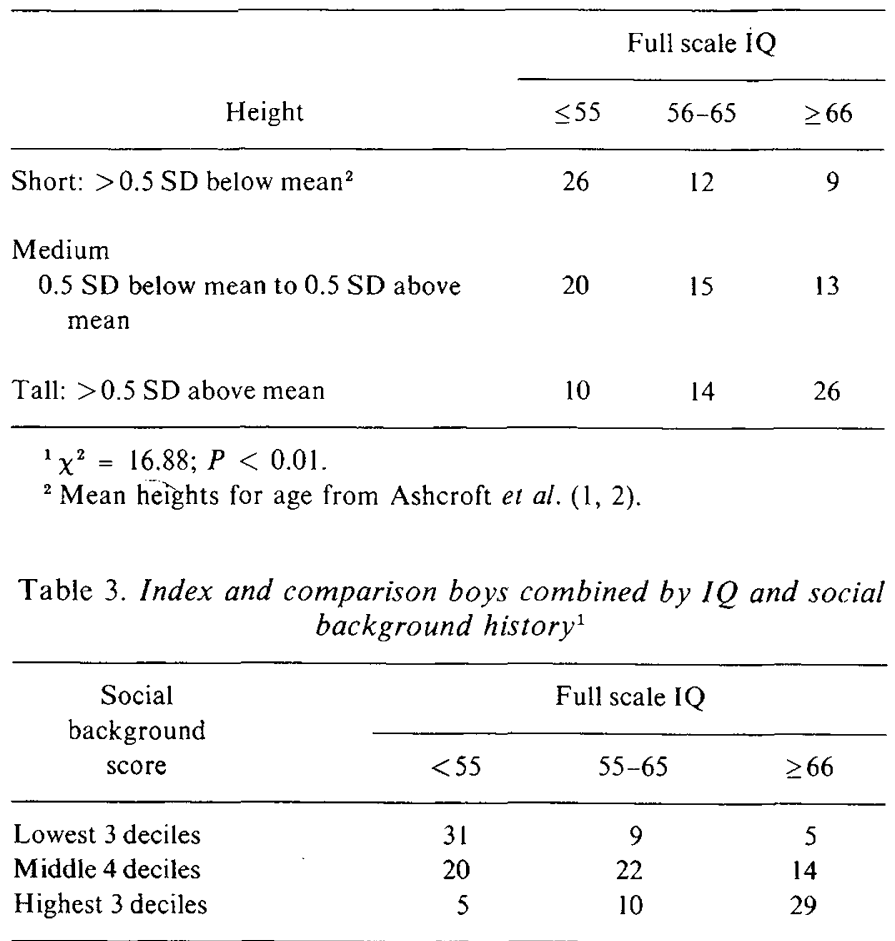

${ }^{1} \chi^{2}=45.2 ; P<0.0005$.

Table 4. Relative contributions of social background, malnutrition, and height to $I Q$ variance estimates ${ }^{1}$

\begin{tabular}{lccl}
\hline & $\begin{array}{c}\text { Social } \\
\text { background } \\
\text { score }\end{array}$ & $\begin{array}{c}\text { Mal- } \\
\text { nutri- } \\
\text { tion }\end{array}$ & Height
\end{tabular}

\footnotetext{
$1 r=0.674$
} 
WISC. Only two of the boys in subset 2 overlap in IQ scores with subset 1 .

We wished to examine whether the differences in IQ between the index and comparison boys varied under differing conditions of height and social background. To do this three groups of social background were made in order to control as far as possible for social background within each group. Using deciles 1 $\cdots 4,5$ and 6 , and 7-9, we established three groups in which there were no significant differences found between the social background of index and comparison boys within each group. Chi square and $t$-tests were used to test for differences. The 10th decile, the most advantageous social background, was omitted because it applied to 12 comparison boys and only 2 index boys.

Because height is associated with age and because, for the boys in the study, age might be associated with IQ, we checked and found no association between age and IQ

The mean and median IQ's of index and comparison boys and the number who have different heights and social backgrounds are shown in Table 6 . This table shows that under certain conditions the index boys have higher IQ's than the comparison subjects. For example, index boys who are tall and have an advantageous social background have an average IQ score 11 points higher than comparison boys who are short and have a disadvantageous social background. Table 6 also shows that under the most favorable conditions of being tall and having an advantageous social background, the average IQ of the index boys is only 2 points lower than the comparison boys. Under the most unfavorable conditions of short stature and disadvantageous social backgrounds, the average IQ of the index boys is 9 points lower than the comparison boys. The combinations of advantageous social background and short stature, and of disadvantageous social back-

Table 5. Individual IQ scores for boys with extremes of advantageous and disadvantageous histories

Subset $1^{1}$ IQ scores $46,46,46,46,46$, $46,46,47,48,51$

$53,54,54, \quad 62$

Subset $2^{2}$ IQ scores

$53, \quad 59, \quad 63,64,67,68$,
$68,69,69,69$,
$72,72,76,79$,
$86, \quad 86, \quad 89$,
101,113

${ }^{1}$ Subset 1: Hospitalization for malnutrition during the first 2 years of life. Short: $>0.5$ SD below mean height. Disadvantageous background history: score in lowest 4 deciles.

${ }^{2}$ Subset 2: No hospitalization for malnutrition. Tall: $>0.5 \mathrm{SD}$ above mean height. Advantageous background history: score in top 4 deciles. ground and tall stature show IQ differences between index and comparison cases of 3 and 7 points, respectively. These differences are illustrated in Figure 1.

\section{DISCUSSION}

The results suggest that an acute episode of severe malnutrition in the first 2 years of life has differing consequences for intellectual development depending on the social background as represented by characteristics of the child's guardian, the economic conditions of the household, and the kinds of social experiences of the child. Further, the consequences are influenced by the life history of the child as reflected in his stature at time of follow-up, which provides an indication of his lifetime nutritional history. Favorable social backgrounds and an overall history of good physical growth appear to be more related to intellectual development than an episode of severe malnutrition in the first 2 years of life.

If severe malnutrition in infancy occurs in a context of a life history which is generally favorable for intellectual development, an early acute episode of malnutrition appears to have a negligible effect on intellectual functioning. If, however, the early acute episode of malnutrition occurs in an unfavorable set of circumstances for intellectual development, the early acute malnutrition has a clear relation to later intellectual impairment.

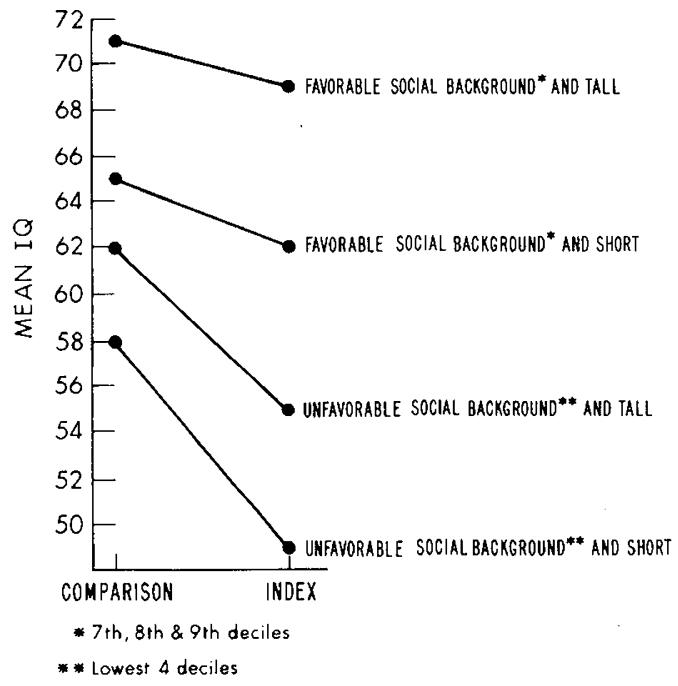

Fig. 1. Mean IQ's of index and comparison of boys when social background and height are held constant.

Table 6. IQ means and medians of index and comparison boys by height and social background ${ }^{1}$

Social background decile

\begin{tabular}{|c|c|c|c|c|c|c|c|c|c|c|c|c|c|c|c|c|c|c|}
\hline \multirow[b]{3}{*}{ Height } & \multicolumn{9}{|c|}{ Index } & \multicolumn{9}{|c|}{ Comparison } \\
\hline & \multicolumn{3}{|c|}{ Lowest 4} & \multicolumn{3}{|c|}{ Middle 2} & \multicolumn{3}{|c|}{$7,8,9$ th } & \multicolumn{3}{|c|}{ Lowest 4} & \multicolumn{3}{|c|}{ Middle 2} & \multicolumn{3}{|c|}{$7,8,9$ th } \\
\hline & $n$ & $\begin{array}{l}\text { IQ } \\
\text { mean }\end{array}$ & $\begin{array}{l}\text { IQ } \\
\text { median }\end{array}$ & $n$ & $\begin{array}{c}\mathrm{IQ} \\
\text { mean }\end{array}$ & $\begin{array}{c}\text { IQ } \\
\text { median }\end{array}$ & $n$ & $\begin{array}{c}\mathrm{IQ} \\
\text { mean }\end{array}$ & $\begin{array}{c}\mathrm{IQ} \\
\text { median }\end{array}$ & $n$ & $\begin{array}{l}\mathrm{IQ} \\
\text { mean }\end{array}$ & $\begin{array}{l}\mathrm{IQ} \\
\text { median }\end{array}$ & $n$ & $\begin{array}{l}\text { IQ } \\
\text { mean }\end{array}$ & $\begin{array}{c}\text { IQ } \\
\text { median }\end{array}$ & $n$ & $\begin{array}{c}\text { IQ } \\
\text { mean }\end{array}$ & $\begin{array}{l}\text { IQ } \\
\text { median }\end{array}$ \\
\hline $\begin{array}{c}\text { Short: }>0.5 \mathrm{SD} \\
\text { below mean }\end{array}$ & 14 & 49 & 47 & 9 & 54 & 54 & 8 & 62 & 57 & 7 & 58 & 58 & 1 & 2 & 2 & 4 & 65 & 65 \\
\hline $\begin{array}{l}\text { Medium: } 0.5 \mathrm{SD} \\
\text { above and below } \\
\text { mean }\end{array}$ & 11 & 52 & 51 & 6 & 58 & 62 & 7 & 67 & 66 & 14 & 55 & 53 & 1 & 2 & 2 & 6 & 69 & 67 \\
\hline $\begin{array}{c}\text { Tall: }>0.5 \mathrm{SD} \\
\text { above mean }\end{array}$ & 8 & 55 & 55 & 4 & 66 & 67 & 6 & 69 & 73 & 6 & 62 & 62 & 7 & 67 & 65 & 13 & 71 & 69 \\
\hline
\end{tabular}

${ }^{1}$ Mean heights for age taken from Ashcroft et al. $(1,2)$.

${ }^{2}$ Indicates lack of sufficient cases. 
It should be emphasized that the Jamaican study applies to boys ages 6-10 who have had high standards of medical care during the acute phase of malnutrition after hospitalization and good nutrition during the period of convalescence after the severe malnutrition. Furthermore, the severe malnutrition occurred during the first 2 years of life. We examined whether the relationship between severe malnutrition and later IQ varied depending on the age at hospitalization within the first 2 years and found no differences by age. Whether different results would have been obtained using girls, different ages at follow-up, and poor conditions of medical care and nutrition during the acute episode and convalescence must be answered in other studies.

The finding that, under disadvantageous circumstances for intellectual development, the index boys have lower IQ's than the comparison boys does not tell us what it was about the episode of severe malnutrition that caused the lower IQ's. The hospitalization may have been associated with other factors which may contribute toward later intellectual impairment, e.g., maternal deprivation.

The results suggest that the explanation that severe malnutrition in infancy causes central nervous system damage which then in turn causes mental retardation or impairment is too simple. A more complex conceptualization is needed which takes into account biologic and social variables that may influence the child's intellectual functioning and development over his lifespan, including the period of fetal growth and the neonatal period. The results reported here only make a start in this direction and will require the addition of further social background variables using a broad human ecologic perspective. It will also require the use of multivariate forms of analysis which take into account the interactions between variables. The only functional outcome or dependent variable reported here was IQ. A broader functional profile of the child is necessary which includes social, emotional, and motor functioning if we are to understand the consequences of severe malnutrition for child development.

\section{SUMMARY}

The IQ's of school boys in Jamaica W.I. aged 6-10 were examined in relation to: $(l)$ whether or not they had experienced an episode of severe malnutrition in the first 2 years of life, (2) their heights at the time of IQ testing, and (3) their social backgrounds using a composite measure of the parent or guardian's level of capability, the economic and housing conditions of the boy's family, and the amount of intellectual stimulation experienced by the boy in early childhood. The presence of severe malnutrition in infancy, small stature, and a disadvantageous social background were each associated separately with low IQ. To examine the relative contribution of the three variables to IQ a multiple correlation coefficient was used. The largest contributor to the variance was the social background measure and the smallest was severe malnutrition. Boys who had experienced severe malnutrition, good physical growth, and a favorable social history had an average IQ 11 points higher than boys who had not been severely malnourished, but had overall poor physical growth and an unfavorable social background. Severe malnutrition in infancy occurring in a context of an overall history of good physical growth and a favorable social background has a negligible effect on intellectual functioning. If, however, severe malnutrition occurs in a context of a poor overall history of physical growth and an unfavorable social background, then the early severe mainutrition, has a clear relation to later intellectual functioning.

\section{REFERENCES AND NOTES}

1. Ashcroft, M. T., and Lovell, H. G.: Heights and weights of Jamaican primary school children. J. Trop. Pediat., 12: 37 (1966).

2. Ashcroft, M. T., Lovell, H. G., and Williams, A.: Heights and weights of infants and children in a rural community of Jamaica. J. Trop. Pediat., 11: 56 (1965).

3. Birch, H. G., Piñeiro, C., Alcade, E., Toca, T., and Cravioto, J.: Relation of kwashiorkor in early childhood and intelligence at school age. Pediat. Res., 5: 579 (1971).

4. Cabak, V., and Najdanvic, R.: Effect of undernutrition in early life on physical and mental development. Arch. Dis. Childhood, 40: 532 (1965).

5. Champakam, S., Srikantia, S. G., and Gopalan, C.: Kwashiorkor and mental development. Amer. J. Clin. Nutr., 21: 844 (1968)

6. Chase, H. P., and Martin, H. P.: Undernutrition and child development. N. Engl. J. Med., 282: 933 (1970).

7. Cravioto, J., and De Licardie, E. R.: Intersensory development of school-age children. In: N. S. Scrimshaw and J. E. Gordon: Malnutrition, Learning and Behavior (M. I. T. Press, Cambridge, Mass. 1968).

8. Cravioto, J., De Licardie, E. R., and Birch, H. G.: Nutrition, growth and neurointegrative development: An experimental and ecologic study. Pediatrics (Suppl.), 38: 319 (1966)

9. Evans, D. E., Moodie, A. D., and Hansen, J. D. L.: Kwashiorkor and intellectual development. S. Afr. Med. J., 45: 1413 (1971)

10. Hertzig, M. E., Birch, H. G., Richardson, S. A., and Tizard, J.: Intellectual levels of school children severely malnourished during the first two years of life. Pediatrics, 49: 814 (1972).

11. Pollitt, E., and Granoff, D.: Mental and motor development of Peruvian children treated for severe malnutrition. Rev. Interamer. Psicol., l: 93 (1967).

12. Richardson, S. A.: Ecology of malnutrition: Non-nutritional factors influencing intellectual and behavioral development. In: Nutrition, the Nervous System and Behavior (Pan American Health Organization, Publication no. 251, 1972).

13. Richardson, S. A.: The background histories of school children severely malnourished in infancy. Advan. Pediat., 21: 167 (1974).

14. Richardson, S. A.: Physical growth of Jamaican school children who were severely malnourished before two years of age. J. Biosoc. Sci. 7: 445 (1975).

15. Richardson, S. A.: The ecology of severe malnutrition and intellectual development. In: D. A. A. Primrose: Proceedings of the Third Congress of the International Association for the Study of Mental Deficiency, Vol. 1 (Polish Medical Publishers, Warsaw, 1975).

16. Richardson, S. A., Birch, H. G., Grabie, E., and Yoder, K.: The behavior of children in school who were severely malnourished in the first two years of life. J. Heaith Soc. Behav., 13: 276 (1972).

17. Richardson, S. A., Birch, H. G., and Hertzig, M. E.: School performance of children who were severely malnourished in infancy. J. Ment. Def., 77: 623 (1973).

18. Richardson, S. A., Birch. H. G., and Ragbeer, C.: The behavior of children at home who were severely malnourished in the first two years of life. J. Biosoc. Sci., 7: 255 (1975).

19. Stoch, M. B., and Smythe, P. M.: Does undernutrition during infancy inhibit brain growth and subsequent intellectual development? Arch. Dis. Childhood, 38: 546 (1963).

20. Stoch, M. B., and Smythe, P. M.: The effect of undernutrition during infancy on subsequent brain growth and intellectual development. S. Afr. Med. J., 41: 1027 (1967).

21. Vernon, P. E.: Intelligence and Cultural Environment. (Methuen, London, 1969).

22. Informed consent was obtained from the children's guardians before all testing was done.

23. The author wishes to thank Professor J. G. Waterlow, Director of the Tropical Metabolism Research Unit, Dr. W. E. Miall, Director of the Epidemiological Research Unit, and his colleagues, Dr. M. Ashcroft and Mrs. P. Desai. The Ministry of Education aided the research by granting permission to study the children in schools. Gathering the data were Nurses Ragbeer, Clark, Gilpin and Inniss. Ellen Grabie and Helene Koller have provided valuable assistance in the analysis. The overall study was planned and carried out in collaboration with Dr. Herbert Birch who was in the Department of Pediatrics and a Senior Investigator at the Kennedy Center, Albert Einstein College of Medicine. He died in February 1973 and his death was a great loss to his friends and colleagues and to research related to disadvantaged children.

24. Support for this study was provided by the Foundation for Child Development, the Nutrition Foundation, Inc., New York, the National Institutes of Health, National Institute of Child Health and Human Development (HD 00719) (NIH-71-2081), and by the British Medical Research Council.

25. Requests for reprints should be addressed to: S. A. Richardson, Ph.D., Albert Einstein College of Medicine of Yeshiva University, 1300 Morris Park Ave., Bronx, N.Y. 10461 (USA).

26. Accepted for publication September 3, 1975. 